\title{
Shocking Outflows in Seyfert Galaxies: An Imaging Perspective
}

\author{
Richard W. Pogge \\ Department of Astronomy, The Ohio State University, $174 \mathrm{~W} .18 \mathrm{th}$ \\ Ave, Columbus, OH 43210-1106, USA
}

\begin{abstract}
Representative results of a search for structures suggestive of shocked gas in the circumnuclear regions of Seyfert galaxies are presented. At issue is whether we can identify regions that appear to be shocked by outflows from the active nucleus. Such regions will be good targets for future spectrophotometric studies to determine what rôle shocks play in the physics of the AGN/host-galaxy interaction.
\end{abstract}

\section{Introduction}

There has been much debate at this colloquium over the importance of shocks in AGNs. Specifically, is the physics (ionization, heating, etc.) of the narrow-line region and the spatially extended emission-line regions dominated by shocks or merely aided and abetted by them? Shocks are often invoked to help explain various anomalies relative to the predictions of pure photoionization models that are observed in the temperature, kinematics, and ionization state of the extended emission-line gas, although they are not the only explanations available. Further, one expects shocks to play some rôle, since shocks are practically ubiquitous in outflow sources seen at more modest scales in our own Galaxy, admittedly with varying degrees of energetic importance. An excellent review of the basic issues may be found in Morse et al. (1996), as well as papers in these proceedings (especially by Viegas, Wilson, Evans, Allen, and Bicknell).

I want to step back a little from the theoretical issues and ask the question: Is there any morphological evidence of shocked gas in Seyfert galaxies? In particular, does imaging reveal any gas structures within the circumnuclear regions of AGNs where we might suspect that shocks play a rôle, whether as the principal ionization source, or as an agent for organizing gas that is otherwise primarily photoionized by the nucleus into the shapes that we see?

\section{Search Strategy}

In outline, the strategy is to start with the extensive ground-based emission-line imaging and spectroscopic surveys of Seyfert nuclei (e.g., Pogge 1989; Mulchaey et al. 1996; Whittle 1992a,b), and select objects based on suggestive emissionline morphologies or evidence of disturbed kinematics. By 'suggestive morphologies' I mean ionized gas regions that resemble in form (if not in scale) the shocked regions well-known from Galactic stellar bipolar outflow sources (e.g., 
bowshocks and jet shocks as seen in Herbig-Haro objects) and supernova remnants. Jet shocks might be expected if the radio jets seen in some Seyferts entrain surrounding material. Bowshocks are expected to form where nuclear outflows ram into the surrounding ISM, and cooling-length arguments suggest that they should be geometrically thin and edge-brightened, with their thicknesses unresolved even at $H S T$ resolution. As such, relatively 'sharp' features in ground-based images should become even sharper in $H S T$ images, and emerge in higher contrast against the diffuse starlight or emission-line gas backgrounds in which we expect them to be embedded. The second step, then, has been to collect archival HST images of galaxies selected out of the ground-based surveys, giving preference to FOC/COSTAR or WFPC2 images (to avoid the difficulties of image reconstruction).

Such a 'sample' is of course fairly biased, and does not constitute a survey for shocked structures per se. Indeed, it is important to emphasize that in general the collection of Seyferts imaged thus far by HST is strongly biased towards those galaxies previously suspected of being 'interesting' by various investigators for a variety of reasons, and thus no statistical conclusions may be drawn from these data in any event.

A surprise from the archival $H S T$ imaging data examined thus far has been how useful the broad-band images are for this search, contrary to my prejudice that narrow-band filter images are best. In a number of cases, sharply defined, high surface-brightness emission regions appear in relatively high contrast against the stellar background of the host galaxies in broad ( $>1000 \AA$ ) filter images (see Fig. 2). A further important benefit of these images is that any dust structures or spiral arms continuing to curl in towards the nucleus proper are readily visible, whereas such features are usually all but lost in narrow-band images. This greatly expands the set of galaxies we may consider, as there is much more broad-band imaging available compared to narrow-band imaging (in consequence of the fact that the restricted set of narrow-band filters available on $H S T$ limits which objects may be studied in this way).

\section{Representative Results}

I show here three examples representative of the types of shock-like morphologies found among the Seyferts studied. These are the best cases found thus far.

Markarian 3: This galaxy, studied in detail by Capetti et al. (1995, 1996), shows the best example seen thus far of a jet shock. The narrow radio jet (Kukula et al. 1993) lies precisely along the axis of the 'bar' of emission-line clouds in the inner $1^{\prime \prime}$ (Fig. 1). No electronic version of the radio map was available for plotting here, but see Capetti et al. for a contour map overlay.

Markarian 573: The relation between the $6 \mathrm{~cm}$ radio continuum structure (Ulvestad \& Wilson 1984) and the bowshock-like emission-line structure has been shown by Pogge \& DeRobertis (1995) and Capetti et al. (1996). In Fig. 2 (left), a WFPC2 image in a filter including [O III] is shown after subtraction of a broad $I$-band (F814W) image. This 'continuum' image over-subtracts starlight in regions of dust extinction, resulting in 'negative' features at dust lanes which 


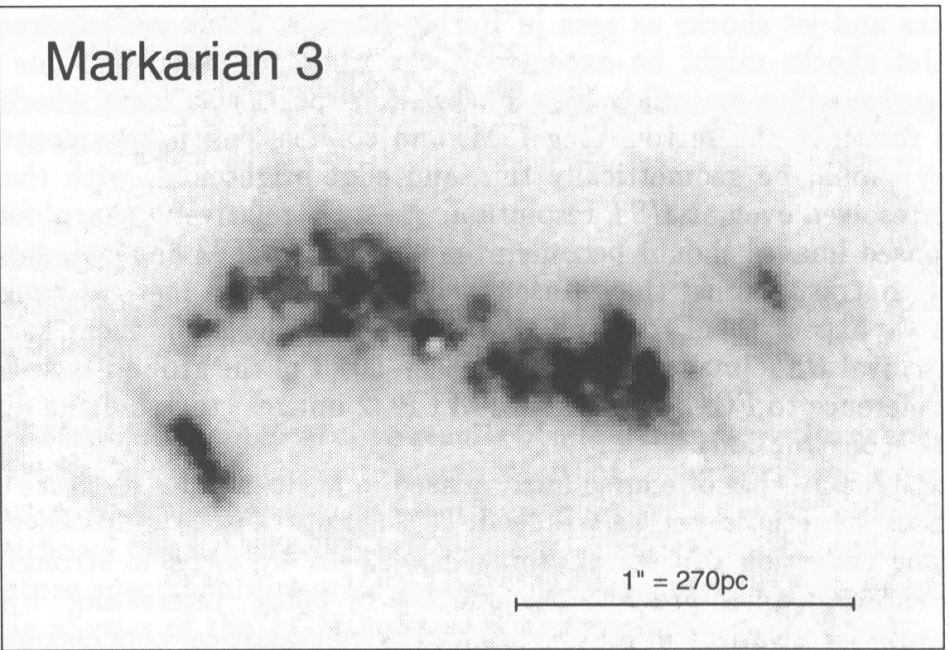

Figure 1. A representative jet shock morphology: FOC (COSTAR) [O III] image of Mrk 3. The MERLIN radio jet map follows the central bar of emission-line clouds (not shown). The nucleus is partly blocked by a Riseau mark. See Capetti et al. (1995 \& 1996) for a detailed discussion.

map to white colors. The radio nucleus is located (by physically well-motivated fiat, not astrometry) at the center of the nuclear spiral dust lanes, not the [O III] peak. This is probably the best example of an optical emission-line bowshock in a Seyfert (the best radio bowshock is in NGC 1068; Wilson \& Ulvestad 1987).

NGC 3393: Figure 2 (right) shows a WFPC2 F606W image of the central $10^{\prime \prime}$. The nucleus is at the apex of dusty nuclear spiral arms, and the S-shaped emission-line structure follows these same arms. The bowshock-like arcs are reminiscent of those in Mrk 573 (left) and of similar scale, but their relation to the spiral dust lanes makes it as likely that we are seeing dense arm gas and dust illuminated by ionizing photons emerging from the active nucleus.

\section{Summary}

The imaging presented here shows representative examples of structures that satisfy our basic notions of what regions of shocked gas should look like. We see structures suggestive of both jets of material in the outflow itself, and bowshocks where it appears that nuclear outflows are impinging on the surrounding interstellar medium, although NGC 3393 has suspiciously located dust features that make interpretation of its arcs as bowshocks less secure. There is, however, forthcoming unpublished work (Baldwin, Lawrence, private comm.) that suggests it may really be a close cousin of Mrk 573, so it is yet not clear how much the local environment can serve to confound our morphological judgment. 


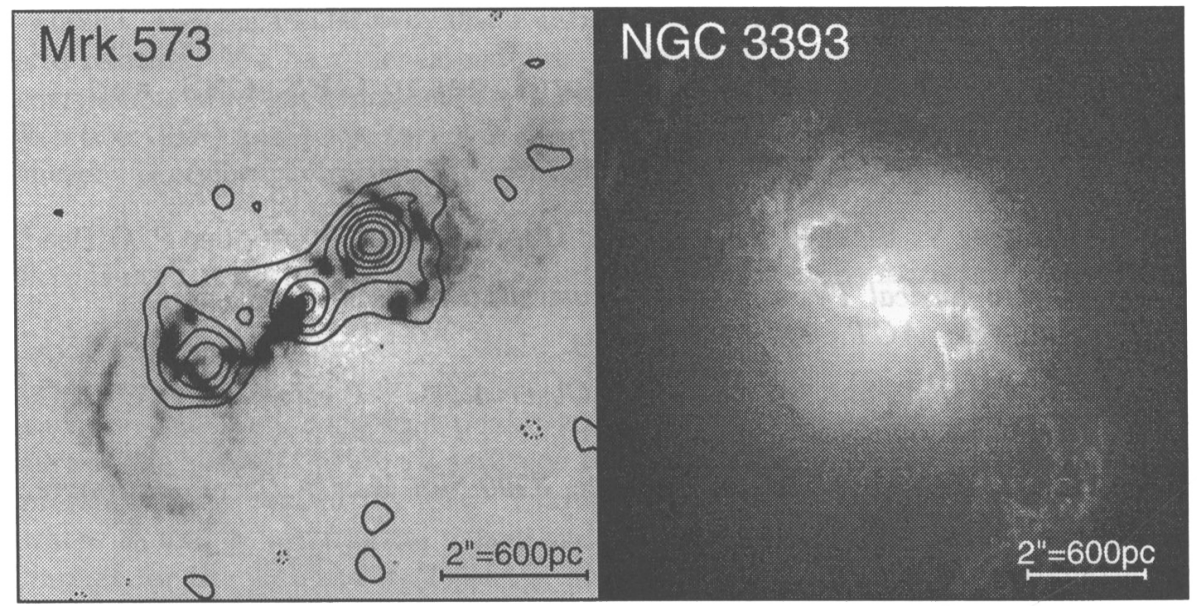

Figure 2. Bowshock-like morphologies: Left: Mrk 573: continuum subtracted WFPC2 [O III] image with VLA $6 \mathrm{~cm}$ radio contours superimposed. Emission clouds are dark and dust features appear white. Right: NGC 3393: WFPC2 F606W image, shown with emission white to improve contrast. The scales are indicated in each panel.

Are there shocks in Seyferts? I believe the answer is 'yes'. Do these shocks do more than sculpt the circumnuclear gas? This is a question that only detailed spectrophotometry to look for tell-tale ionization and kinematic signatures will address. Whether these spectra will provide definitive answers remains to be seen, a conclusion which should come as a shock to nobody.

Acknowledgments. Support for this work was provided by NASA through grant number AR-06380.01-95A from the Space Telescope Science Institute, which is operated by the Association of Universities for Research in Astronomy, Inc., under NASA contract NAS5-26555.

\section{References}

Capetti, A., et al. 1995, ApJ, 448, 600.

Capetti, A., et al. 1996, ApJ, 469, 554.

Kukula, M., et al. 1993, MNRAS, 264, 893.

Morse, J. A., Raymond, J. C., \& Wilson, A.S. 1996, PASP, 108, 426.

Mulchaey, J.S., Wilson, A.S., \& Tsvetanov, Z.I. 1996, ApJS, 102, 309.

Pogge, R. W. 1989, ApJ, 345, 730.

Pogge, R.W. \& DeRobertis, M. M. 1995, ApJ, 451, 585.

Ulvestad, J. S., \& Wilson, A. S. 1984, 278, 544.

Whittle, M. 1992a, ApJ, 387, 109.

Whittle, M. 1992b, ApJ, 387, 121.

Wilson, A.S., \& Ulvestad, J.S. 1987, ApJ, 319, 105. 